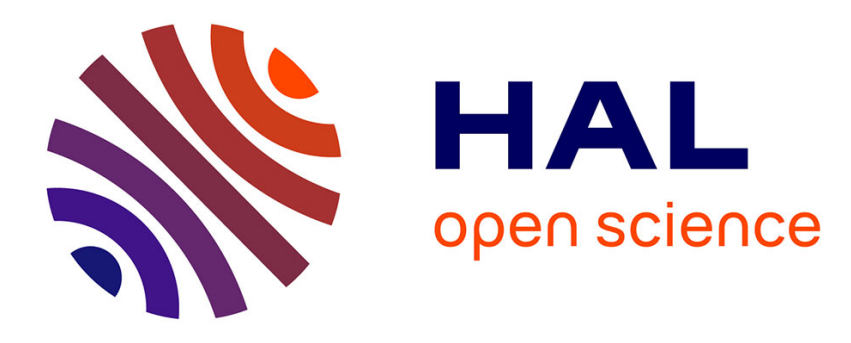

\title{
Arginine supplementation and cardiometabolic risk
}

François Mariotti

\section{To cite this version:}

François Mariotti. Arginine supplementation and cardiometabolic risk. Current Opinion in Clinical Nutrition and Metabolic Care, 2020, 23 (1), pp. 29-34. 10.1097/MCO.0000000000000612 . hal02346025

\section{HAL Id: hal-02346025 \\ https://hal.science/hal-02346025}

Submitted on 20 Sep 2020

HAL is a multi-disciplinary open access archive for the deposit and dissemination of scientific research documents, whether they are published or not. The documents may come from teaching and research institutions in France or abroad, or from public or private research centers.
L'archive ouverte pluridisciplinaire HAL, est destinée au dépôt et à la diffusion de documents scientifiques de niveau recherche, publiés ou non, émanant des établissements d'enseignement et de recherche français ou étrangers, des laboratoires publics ou privés. 
3 This is not the final published version.

4 Full reference: Mariotti, F. Arginine supplementation and cardiometabolic risk. Current Opinion in

5 Clinical Nutrition and Metabolic Care: January 2020 - Volume 23 - Issue 1 - p 29-34. doi:

6 10.1097/MCO.0000000000000612.

7

8 
Title of review article: Arginine supplementation and cardiometabolic risk

\section{Author(s): François Mariotti}

Authors' affiliations: UMR PNCA, AgroParisTech, INRA, Université Paris-Saclay, 75005, Paris, France.

\section{Author of correspondence:}

Name: Prof. François Mariotti

Address: UMR PNCA, AgroParisTech, 16 rue Claude Bernard, F-75005, Paris, France.

Telephone number: none that I want to be public - thank you.

Email address: $\underline{\text { francois.mariotti@agroparistech.fr }}$

\section{$\underline{\text { Abstract }}$}

Purpose of review: Because arginine is the substrate for nitric oxide synthesis, which is pivotal to vascular homeostasis and linked to the insulin response, it has long been posited that supplemental arginine could benefit cardiometabolic health.

Recent findings: Recent data have supported the view that supplemental arginine could alleviate the initiation and development of endothelial dysfunction and also shown that it may reduce the risk of type 2 diabetes. One important finding is that these effects may indeed vary as a function of the amount of arginine, its form and notably the metabolic status of the population. Some studies have shown that low doses of slow-release arginine are better used for NO synthesis and beneficial in individuals with abnormal arginine metabolism/bioavailability. Pathophysiological data in rodents have emphasized the importance of arginase activation during the development of cardiometabolic risk, which lends credence to a potential benefit for arginine supplements. Likewise, epidemiological evidence suggests that alterations to arginine bioavailability are important regarding the cardiometabolic risk. However, other metabolic mechanisms linked to the multiple pathways of arginine metabolism may also play a role.

Summary: Further studies are needed to confirm and analyze how and when supplemental arginine is beneficial to cardiometabolic health.

Keywords: L-arginine; arginine metabolism; Endothelial function; insulin resistance; nitric oxide. 
38 Abbreviations: NO, nitric oxide 


\section{(1)}

\section{Introduction:}

Since the discovery that arginine was the unique substrate for the synthesis of nitric oxide (NO) which plays a critical and pivotal role in vascular homeostasis, it has been suggested that supplemental arginine might be beneficial in alleviating or preventing atherosclerosis. Then, because of growing recognition of the association between insulin sensitivity and endothelial function [1] and the possible role that NO might play in systemic alterations to metabolic fluxes and hemodynamics [2], it has also been proposed arginine could have potential benefits on a broad cluster of metabolic abnormalities that constitute cardiometabolic risk factors which increase the risks of cardiovascular disease and type 2 diabetes.

In this review, we will particularly focus on the most recent findings regarding the effects of supplemental arginine on preventing or alleviating risk factors such as endothelial dysfunction and insulin resistance. We will not present data on arginine supplementation on other endpoints, or in specific populations, although these make the case for its metabolic impact, such as recent findings regarding preeclampsia in animal models [3, 4] and clinical trials [5]. The review will focus on data in humans but also report findings from animal studies useful to understand the possible mechanisms. We will not directly consider data from cellular experiments that would not take account of the fundamental compartmentation of the arginine nutritional system e.g. [6, 7]. Nor will we consider studies where arginine supplementation has been combined with that of other nutrients $[8,9]$ because it is not possible to disentangle the specific role of arginine.

\section{Endothelial function}

Recent reviews and meta-analyses have supported the view that supplemental arginine favors endothelial function in humans $[10,11,12 *]$. However, Rodrigues-Krause et al. reported that the results were highly heterogeneous and that the positive effects of arginine supplementation on endothelial function (evaluated as flow-mediated dilation) could only be identified by reducing heterogeneity in order to discard extreme results [12*].

Those findings have also been supported by animal studies using diet-induced cardiometabolic risk. For instance, in fructose-fed rats, supplemental arginine prevented the impairment of endotheliumdependent vasodilation and hyper-reactivity to phenylephrine, to the same degree as aerobic training [13]. Both treatments increased plasma nitrite, indicative of increased eNOS activity. 
In a randomized double-blind trial in healthy humans with elevated triglycerides and enlarged waist, we found that supplemental arginine (1.5g 3 times/d) did not improve fasting endothelial function but lowered postprandial dysfunction in individuals with lower baseline plasma arginine concentrations $\left[14^{*}\right]$. This finding was in line with an earlier report that a moderate amount of arginine $(2.8 \mathrm{~g})$ added to a high-fat meal could specifically prevent postprandial endothelial dysfunction [15]. Meta-analyses and clinical trials have documented that arginine might have beneficial effects at levels of intake that could be manipulated by the diet (i.e. addition of $3-6 \mathrm{~g} / \mathrm{d}$ to an average baseline dietary intake of $\sim 5 \mathrm{~g} / \mathrm{d})[16,17]$ in individuals displaying an impairment of baseline endothelial function [17]. In the elderly, arginine (3g) rescued the impairment in femoral blood flow and muscle microvascular blood volume in response to $15 \mathrm{~g}$ amino acids [18]. In healthy young individuals, we would expect that the effect of moderate doses of arginine would mainly be seen in those with baseline metabolic/physiological alterations and/or during an allostatic challenge, such as that elicited by a high saturated fat meal.

\section{Insulin sensitivity and the diabetes risk}

In 2018, Monti and collaborators reported their analysis of 90 months of follow-up of an 18-month, randomized, double-blind trial of arginine supplementation $(6.4 \mathrm{~g} / \mathrm{d})$ in 144 individuals with a cardiometabolic risk [19**]. The authors found a $34 \%$ lower risk of becoming diabetic in the arginine supplemented group, indicating the permanence of their findings at 12 months of follow-up. Another important study recently reported a beneficial effect of supplemental arginine on insulin sensitivity. In a randomized cross-over study, overweight men with prediabetes (i.e. elevated baseline plasma glucose or postprandial glucose intolerance) received $9 \mathrm{~g} / \mathrm{d}$ arginine or placebo [20**]. In men of European descent (but not those from southern Asian), arginine supplementation improved insulin sensitivity and glucose control (as assessed by an oral glucose tolerance test). This was concomitant with a marked increase in cold-induced glucose oxidation in men of European descent only. As with endothelial function, these findings have been supported by data in rats [13] and earlier findings in humans at moderate doses of arginine [16].

These important results in humans echo a recent analysis of data from the PREDIMED trial. Using a case-cohort design, the authors estimated the relationship between changes to plasma arginine during the trial in 892 individuals at high cardiovascular risk [21*]. They found a negative association between changes to plasma arginine and the risk of incident type 2 diabetes (-29\% risk per 1 SD increment). However, it remains unclear whether the complex changes in arginine-related metabolites reflect changes to arginine intake or to arginine metabolism, particularly with regards to arginine availability for NO synthesis. 
104

105

106

107

108

109

110

111

112

113

114

115

116

117

118

119

120

121

122

123

124

125

126

127

128

129

130

131

132

133

The effect of supplemental arginine on blood pressure were further documented by a wellconducted, large, randomized single-blind trials in Iran where 90 individuals with obesity or visceral obesity were supplemented with arginine at either $1 \mathrm{~g} 3$ times/d or $2 \mathrm{~g} 3$ times/d, or given a placebo [22*]. Systolic blood pressure decreased by $4 \mathrm{mmHg}$ and $8 \mathrm{mmHg}$ with the $3 \mathrm{~g}$ and $6 \mathrm{~g}$ supplementation doses, respectively. Arginine supplementation also resulted in body weight loss (3\% and $9 \%)$, which could explain part of the important reduction in blood pressure [22*].

Although it could have been driven by changes to body weight, this reduction in blood pressure was in line with most evidence in this regard. Notably, in 2011, the meta-analysis by Dong et al. concluded that arginine supplementation reduced systolic blood pressure by $\sim 5 \mathrm{mmHg}$ and diastolic blood pressure by $\sim 3 \mathrm{mmHg}[10,23]$. Comparing the very high doses of $30 \mathrm{~g}$ and $15 \mathrm{~g}$ arginine supplements to a placebo in overweight/obese healthy individuals, McNeal also reported that $30 \mathrm{~g} / \mathrm{d}$ lowered systolic blood pressure (by $\sim 5 \mathrm{mmHg}$ ), in female participants only [24*].

The effects of arginine supplementation on body weight in the study by Dashtabi et al had already been reported by some previous trials and animal studies [10]. In my opinion, the data remain inconclusive in humans. Possible mechanisms include a reduced food intake (presumably by a direct central mechanism rather than mediated by the stimulation of gut hormone release, as recently proposed [25]), and changes to adipose tissue metabolism [26]. Arginine does not appear to affect the thermoregulatory response to stress in humans [27].

Few studies have reported effects of arginine supplementation on inflammatory pathways. Using moderate amounts of arginine to supplement the diet of rats, Hong et al. recently reported downregulation of nuclear factor kappaB p65 and a lowering of plasma CRP [28]. Kim et al. [29] also found that low amount of arginine supplementation reduced NF-KB expression in exercising rats fed a high-fat diet, and also reduced TNFa in rats fed a high-fat diet.

\section{At which dose, in which form and for which people?}

The first studies of arginine supplementation were conducted at relatively high doses (mostly 9$12 \mathrm{~g} / \mathrm{d}$ ). However, some studies have investigated the potential of lower doses, commensurable with spontaneous dietary intake and its possible variation resulting from changes to the level and type of dietary protein consumed. Studies at low doses are important because the favorable effects of some foods are often ascribed, at least in part, to the amount of arginine they contain - e.g. nuts [30, 31]. As discussed above, the effects of low-dose arginine on endothelial function and insulin sensitivity 
have been reported at low doses in clinical trials $\left[14^{*}, 15,16\right]$ and in the positive meta-analysis conducted by Bai et al., no relationship with the dose was evidenced [17].

Because dietary arginine is consumed naturally as dietary protein, it is made available much more slowly than the free arginine (i.e. arginine chlorhydrate or Arginine base) found in supplements. It has been proposed that the speed of absorption could impact the metabolic utilization of supplemental arginine, as is often the case for amino acids and nutrients in general. Using labeled arginine in a cross-over trial, we evidenced that arginine in sustained-release form was directed more to NO synthesis than arginine in the immediate release form [32*]. In this trial we also found that the utilization of supplemental arginine for NO synthesis was higher in individuals with cardiometabolic risk factors than in control subjects, thus offering the first observation at the metabolic level explaining that the benefits of arginine supplementation have mostly been demonstrated in individuals with cardiometabolic risk factors and altered baseline NO-related functions.

Against this background, low doses of sustained-release arginine have indeed been reported to exert favorable effects on endothelial function and insulin resistance in individuals with cardiometabolic risk factors $\left[14^{*}, 16,33\right]$. Recent studies in rats could be considered as contributing little to the topic because they were mostly conducted at doses (between 0.9 and $2.5 \mathrm{~g} / \mathrm{kg} \mathrm{bw}$ ) that are higher than what could be achieved in humans, even if the arginine was given by enriching the food or water, thus spreading delivery over the wakening period [13, 34, 35, 36*].

Conversely, it has long been suggested that supplemental arginine might be detrimental in certain situations of overt disease because NO synthesis may mostly be operating by the inducible form of NO synthase (iNOS) which generates oxidative and nitrosative stress. In this situation, supplemental arginine might indeed fuel this detrimental process. This was first proposed many years ago [37] and recently further illustrated by the finding that an increase in iNOS activity and the resulting nitrosative stress was pivotal in a model of heart failure [38]. The benefits of arginine supplement may therefore vary depending on the degree of activation of iNOS over constitutive NOS isoform (eNOS), and the reorganization of NO synthesis regulation during the development of obesity and/or associated cardiometabolic risks [37, 39, 40]. Nonetheless, the detrimental effects of arginine supplements may be limited to these specific situations of acute disease, and arginine supplementation has been documented as being safe in a wide variety of situations $[10,41,42]$.

\section{Reduced arginine bioavailability in a context of cardiometabolic risk}

There is still widespread agreement that the development of cardiometabolic risk is associated with a reduction in NO synthesis [43], resulting from alterations to the metabolism of arginine and its bioavailability for NO synthesis [21]. However, no good markers for arginine bioavailability yet exist 
in humans, so it is mostly studied from the ratio of arginine to other metabolites (ornithine and citrulline or methylated arginine) in fasting plasma, whereas arginine metabolism is highly compartmented and plasma arginine is not the metabolic precursor of NO synthesis [44].

Animal models have been of value to understanding the alterations to arginine metabolism that operate during the onset and development of obesity and cardiometabolic risk, and have shown that increased arginase activity may be the key to reducing NO synthesis-. In a well-conducted study using high-fat high-sucrose diets in rodents and endothelial cells culture, Bhatta et al. showed that the deletion or inhibition of arginase activity prevents endothelial dysfunction, arterial rigidity, the impairment of endothelial cell NO synthesis and increases in oxidative and nitrosative stress, as well as preserving a normal ratio of arginine over ornithine and citrulline [45**]. Other studies have recently highlighted the role of arginase in the development of endothelial function, and notably the importance of the up-regulation of arginase 1 in red blood cells, secondary to an increase in oxidative stress [46]. It has also been shown that perivascular adipose tissue could also contribute to dietrelated endothelial dysfunction by the induction of arginase [47]. An increase in asymmetric dimethyl arginine is still thought to be implicated in endothelial dysfunction and insulin resistance, which may operate by the competitive inhibition of NO synthesis [48]. Taken together, these findings therefore indicate that an increase in I-arginine availability (e.g. by arginine administration or the inhibition of arginase) results in the prevention of cardiometabolic abnormalities $[46,47]$ which remains strongly compatible with the potential of supplemental arginine to provide a supplemental substrate to overcome or alleviate the reduction in NO synthesis.

In a more novel manner, recent studies have suggested that arginine supplementation could affect the microbiota and intestinal epithelial functions, which might ultimately modulate systemic proinflammatory conditions [49]. Recently, the attenuation of fructose-induced non-alcoholic steatohepatitis using supplemental arginine was shown to be accompanied by an alleviation of decreased tight junction protein levels in the upper portions of the small intestine, of increased levels of bacterial endotoxins in portal plasma and a rise in hepatic toll-like receptor-4 mRNA [36]. The precise mechanism still needs to be elucidated.

Supplemental arginine may have a physiological and pathophysiological impact that might originate from its effects on the many pathways other than NO synthesis. In recent years, numerous studies have been performed on homoarginine, a new player in the area of arginine and the cardiometabolic risk. Homoarginine has been proposed as a protective risk factor for cardiovascular disease and diabetes [50], although the underlying mechanism remains highly unclear [51*]. Importantly, 
199 arginine administration has been reported to increase homoarginine synthesis in various models

200 including rats, pigs and humans in the acute and chronic settings [52, 53].

\section{Conclusion}

202 There is now further evidence regarding the benefits of supplemental arginine during the initiation 203 and development of cardiometabolic risk, namely with respect to endothelial dysfunction and the 204 risk of type 2 diabetes. These benefits may indeed vary as a function of the amount of arginine, its 205 type and notably the metabolic status of the population. Pathophysiological data and 206 epidemiological findings have highlighted abnormalities in arginine bioavailability that lend credence 207 to a potential benefit for arginine supplements. Further studies are now needed to confirm and 208 analyze how and when supplemental arginine could be beneficial to cardiometabolic health. 
Key points:

211 (3-5 key points/sentences that summarize your article)

- There is now more evidence that supplemental arginine may benefit endothelial dysfunction and insulin sensitivity, and possibly other cardiometabolic risk factors.

- The benefits may vary depending on its dose and type, and notably the metabolic status of the population.

- Supplemental arginine may alleviate the development of the arginine metabolism abnormalities that constitute important background mechanisms for increases in the cardiometabolic risk.

- Further studies could interestingly address variations in the effects of supplemental arginine as a function of the background arginine metabolism of individuals.

\section{Financial support and sponsorship: none.}

\section{Conflicts of interest: None.}


[1] Li M, Qian M, Xu J. Vascular Endothelial Regulation of Obesity-Associated Insulin Resistance. Front Cardiovasc Med 2017; 4:51.

[2] Bryan NS. Functional Nitric Oxide Nutrition to Combat Cardiovascular Disease. Current atherosclerosis reports 2018; 20:21.

[3] Arikawe AP, Udenze IC, Olusanya AW et al. L-arginine supplementation lowers blood pressure, protein excretion and plasma lipid profile in experimental salt-induced hypertension in pregnancy: Relevance to preeclampsia. Pathophysiology : the official journal of the International Society for Pathophysiology 2019. suppression of angiogenic risk factors in a rat model of preeclampsia. Pathophysiology : the official journal of the International Society for Pathophysiology 2018; 25:389-395.

[5] Tenorio MB, Ferreira RC, Moura FA et al. Oral antioxidant therapy for prevention and treatment of preeclampsia: Meta-analysis of randomized controlled trials. Nutr Metab Cardiovasc Dis 2018; 28:865-876.

[6] Zheng $H$, Guo Q, Duan X et al. I-arginine inhibited apoptosis of fish leukocytes via regulation of NFkappaB-mediated inflammation, NO synthesis, and anti-oxidant capacity. Biochimie 2019; 158:62-72. [7] Tsuboi T, Maeda M, Hayashi T. Administration of L-arginine plus L-citrulline or L-citrulline alone successfully retarded endothelial senescence. PloS one 2018; 13:e0192252.

[8] Menzel D, Haller H, Wilhelm M, Robenek H. L-Arginine and B vitamins improve endothelial function in subjects with mild to moderate blood pressure elevation. Eur J Nutr 2018; 57:557-568. [9] Ellis AC, Patterson M, Dudenbostel T et al. Effects of 6-month supplementation with betahydroxy-beta-methylbutyrate, glutamine and arginine on vascular endothelial function of older adults. European journal of clinical nutrition 2016; 70:269-273.

[10] McNeal CJ, Meininger CJ, Reddy D et al. Safety and Effectiveness of Arginine in Adults. The Journal of nutrition 2016; 146:2587s-2593s.

[11] Hu S, Han M, Rezaei A et al. L-Arginine Modulates Glucose and Lipid Metabolism in Obesity and Diabetes. Current protein \& peptide science 2017; 18:599-608.

[12] Rodrigues-Krause J, Krause M, Rocha I et al. Association of I-Arginine Supplementation with Markers of Endothelial Function in Patients with Cardiovascular or Metabolic Disorders: A Systematic Review and Meta-Analysis. Nutrients 2018; 11.

* An important meta-analysis which showed that the positive effect of arginine supplementation on endothelial function (evaluated as flow-mediated dilation) could only be identified when reducing heterogeneity by discarding extreme results.

[13] Medeiros RF, Gaique TG, Bento-Bernardes T et al. Arginine and aerobic training prevent endothelial and metabolic alterations in rats at high risk for the development of the metabolic syndrome. Br J Nutr 2017; 118:1-10.

[14] Deveaux A, Pham I, West SG et al. I-Arginine Supplementation Alleviates Postprandial Endothelial Dysfunction When Baseline Fasting Plasma Arginine Concentration Is Low: A Randomized Controlled Trial in Healthy Overweight Adults with Cardiometabolic Risk Factors. The Journal of nutrition 2016; 146:1330-1340.

* Confirmation of the postprandial modulation of endothelial dysfunction by supplemental arginine at relatively low doses. In addition, evidence that the effect may be beneficial in individuals with altered baseline plasma arginine availability.

[15] Borucki K, Aronica S, Starke I et al. Addition of $2.5 \mathrm{~g} \mathrm{L-arginine} \mathrm{in} \mathrm{a} \mathrm{fatty} \mathrm{meal} \mathrm{prevents} \mathrm{the}$ lipemia-induced endothelial dysfunction in healthy volunteers. Atherosclerosis 2009; 205:251-254. 
[16] Monti LD, Casiraghi MC, Setola E et al. L-arginine enriched biscuits improve endothelial function and glucose metabolism: a pilot study in healthy subjects and a cross-over study in subjects with impaired glucose tolerance and metabolic syndrome. Metabolism 2013; 62:255-264.

[17] Bai Y, Sun L, Yang T et al. Increase in fasting vascular endothelial function after short-term oral Larginine is effective when baseline flow-mediated dilation is low: a meta-analysis of randomized controlled trials. The American journal of clinical nutrition 2009; 89:77-84.

[18] Mitchell WK, Phillips BE, Wilkinson DJ et al. Supplementing essential amino acids with the nitric oxide precursor, l-arginine, enhances skeletal muscle perfusion without impacting anabolism in older men. Clinical nutrition (Edinburgh, Scotland) 2017; 36:1573-1579.

[19] Monti LD, Galluccio E, Villa V et al. Decreased diabetes risk over 9 year after 18-month oral Larginine treatment in middle-aged subjects with impaired glucose tolerance and metabolic syndrome (extension evaluation of L-arginine study). Eur J Nutr 2018; 57:2805-2817.

** An important report on 90 months of follow-up to an 18-month, randomized, double-blind trial of arginine supplementation $(6.4 \mathrm{~g} / \mathrm{d})$ in 144 individuals with cardiometabolic risk. The authors found a $34 \%$ lower risk of becoming diabetic in the arginine supplemented group, indicating the permanence of this benefit which was initially evidenced at 12 months of follow-up.

[20] Boon MR, Hanssen MJW, Brans B et al. Effect of L-arginine on energy metabolism, skeletal muscle and brown adipose tissue in South Asian and Europid prediabetic men: a randomised doubleblinded crossover study. Diabetologia 2019; 62:112-122.

** In this excellent study, arginine supplements improved insulin sensitivity and glucose control in men of European descent, but not in men from southern Asia. This was concomitant with a marked increase in cold-induced glucose oxidation, in men of European descent only.

[21] Yu E, Ruiz-Canela M, Razquin C et al. Changes in arginine are inversely associated with type 2 diabetes: A case-cohort study in the PREDIMED trial. Diabetes, obesity \& metabolism 2019; 21:397401.

* A case-cohort study which reported a negative association between changes to plasma arginine and the risk of incident type 2 diabetes (-29\% risk per 1 SD increment). It is still unknown whether these changes were the consequences of changes to intake or metabolic changes reflecting changes in bioavailability, but this lends credence to the view that sustaining arginine bioavailability could be important to limiting the risk of diabetes.

[22] Dashtabi A, Mazloom Z, Fararouei M, Hejazi N. Oral L-Arginine Administration Improves Anthropometric and Biochemical Indices Associated With Cardiovascular Diseases in Obese Patients: A Randomized, Single Blind Placebo Controlled Clinical Trial. Research in cardiovascular medicine 2016; 5:e29419.

* In 90 individuals with obesity or visceral obesity who were supplemented with arginine, at either $1 \mathrm{~g}$ three times a day or $2 \mathrm{~g}$ three times a day, or given a placebo, the authors reported reductions in systolic blood pressure of $4 \mathrm{mmHg}$ and $8 \mathrm{mmHg}$, respectively - another study confirming earlier reports. Less common was the finding that arginine supplementation also resulted in body weight loss (3\% and $9 \%)$, which could explain part of the important reduction in blood pressure.

[23] Dong JY, Qin LQ, Zhang Z et al. Effect of oral L-arginine supplementation on blood pressure: a meta-analysis of randomized, double-blind, placebo-controlled trials. American heart journal 2011; 162:959-965.

[24] McNeal CJ, Meininger CJ, Wilborn CD et al. Safety of dietary supplementation with arginine in adult humans. Amino acids 2018; 50:1215-1229.

* Further results of the favorable effects on cardiometabolic risk factors (systolic blood pressure and plasma glucose in women, and plasma free fatty acids) obtained with a high dosage of L-arginine $(30 \mathrm{~g} / \mathrm{d})$. 
[25] Alamshah A, McGavigan AK, Spreckley E et al. L-arginine promotes gut hormone release and reduces food intake in rodents. Diabetes, obesity \& metabolism 2016; 18:508-518.

[26] Atawia RT, Bunch KL, Toque HA et al. Mechanisms of obesity-induced metabolic and vascular dysfunctions. Frontiers in bioscience 2019; 24:890-934.

[27] Tyler CJ, Coffey TR, Hodges GJ. Acute L-arginine supplementation has no effect on cardiovascular or thermoregulatory responses to rest, exercise, and recovery in the heat. Eur J Appl Physiol 2016; 116:363-371. [28] Hong MY, Beidler J, Hooshmand S et al. Watermelon and l-arginine consumption improve serum lipid profile and reduce inflammation and oxidative stress by altering gene expression in rats fed an atherogenic diet. Nutrition research 2018; 58:46-54.

[29] Kim HJ, Son J, Jin E et al. Effects of exercise and L-arginine intake on inflammation in aorta of high-fat diet induced obese rats. J Exerc Nutrition Biochem 2016; 20:36-40.

[30] Bitok E, Sabate J. Nuts and Cardiovascular Disease. Progress in cardiovascular diseases 2018; 61:33-37.

[31] Kim Y, Keogh JB, Clifton PM. Benefits of Nut Consumption on Insulin Resistance and Cardiovascular Risk Factors: Multiple Potential Mechanisms of Actions. Nutrients 2017; 9. [32] Deveaux A, Fouillet H, Petzke KJ et al. A Slow- Compared with a Fast-Release Form of Oral Arginine Increases Its Utilization for Nitric Oxide Synthesis in Overweight Adults with Cardiometabolic Risk Factors in a Randomized Controlled Study. The Journal of nutrition 2016; 146:1322-1329.

* Key background metabolic evidence that supplemental arginine is used more for NO synthesis when the individuals present cardiometabolic risk factors. This study also showed that the potential for supplemental arginine to rescue NO synthesis was greater when arginine was made available more slowly, as it is when provided as protein by the diet.

[33] Boger GI, Rudolph TK, Maas R et al. Asymmetric dimethylarginine determines the improvement of endothelium-dependent vasodilation by simvastatin: Effect of combination with oral L-arginine. Journal of the American College of Cardiology 2007; 49:2274-2282.

[34] Motta NAV, Fumian MM, Medeiros RF et al. Aerobic Training Associated with Arginine Supplementation Reduces Collagen-Induced Platelet Hyperaggregability in Rats under High Risk to Develop Metabolic Syndrome. International journal of endocrinology 2019; 2019:8919435. [35] Peyton KJ, Liu XM, Shebib AR et al. Arginase inhibition prevents the development of hypertension and improves insulin resistance in obese rats. Amino acids 2018; 50:747-754. [36] Sellmann C, Degen C, Jin CJ et al. Oral arginine supplementation protects female mice from the onset of non-alcoholic steatohepatitis. Amino acids 2017; 49:1215-1225.

* This study in rats showed that the attenuation of fructose-induced non-alcoholic steatohepatitis by supplemental arginine was accompanied by an alleviation of impaired intestinal barrier function in the upper portions of the small intestine and low-grade endotoxemia in portal plasma.

[37] Maxwell AJ. Mechanisms of dysfunction of the nitric oxide pathway in vascular diseases. Nitric Oxide 2002; 6:101-124.

[38] Schiattarella GG, Altamirano F, Tong D et al. Nitrosative stress drives heart failure with preserved ejection fraction. Nature 2019; 568:351-356. Promote Endothelial Dysfunction via Increasing Leptin/Akt/eNOS Signaling. Frontiers in physiology 2019; 10:268.

[40] Blouet C, Mariotti F, Mathe V et al. Nitric oxide bioavailability and not production is first altered during the onset of insulin resistance in sucrose-fed rats. Experimental biology and medicine 2007; 232:1458-1464.

[41] Rosenthal MD, Carrott PW, Patel J et al. Parenteral or Enteral Arginine Supplementation Safety and Efficacy. The Journal of nutrition 2016; 146:2594S-2600S.

[42] Wu Z, Hou Y, Hu S et al. Catabolism and safety of supplemental L-arginine in animals. Amino acids 2016; 48:1541-1552. 
[43] Giam B, Kuruppu S, Head GA et al. Effects of Dietary I-Arginine on Nitric Oxide Bioavailability in Obese Normotensive and Obese Hypertensive Subjects. Nutrients 2016; 8.

[44] Mariotti F, Petzke KJ, Bonnet D et al. Kinetics of the utilization of dietary arginine for nitric oxide and urea synthesis: insight into the arginine-nitric oxide metabolic system in humans. The American journal of clinical nutrition 2013; 97:972-979. [45] Bhatta A, Yao L, Xu Z et al. Obesity-induced vascular dysfunction and arterial stiffening requires endothelial cell arginase 1. Cardiovascular research 2017; 113:1664-1676.

** An excellent demonstration in mice that the activation of arginase is crucial to the onset of cardiovascular dysfunction and cardiometabolic abnormalities in diet-induced obesity. This lends credence to the benefits of supplemental arginine to rescue a lower arginine availability.

[46] Zhou Z, Mahdi A, Tratsiakovich Y et al. Erythrocytes From Patients With Type 2 Diabetes Induce Endothelial Dysfunction Via Arginase I. Journal of the American College of Cardiology 2018; 72:769780.

[47] Xia N, Horke S, Habermeier A et al. Uncoupling of Endothelial Nitric Oxide Synthase in Perivascular Adipose Tissue of Diet-Induced Obese Mice. Arteriosclerosis, thrombosis, and vascular biology 2016; 36:78-85. arginase up-regulation contribute to endothelial dysfunction related to insulin resistance in rats and morbidly obese humans. J Physiol 2016; 594:3045-3060. [49] Singh K, Gobert AP, Coburn LA et al. Dietary Arginine Regulates Severity of Experimental Colitis and Affects the Colonic Microbiome. Frontiers in cellular and infection microbiology 2019; 9:66. [50] Karetnikova ES, Jarzebska N, Markov AG et al. Is Homoarginine a Protective Cardiovascular Risk Factor? Arteriosclerosis, thrombosis, and vascular biology 2019; 39:869-875.

[51] Tsikas D, Bollenbach A, Hanff E, Kayacelebi AA. Asymmetric dimethylarginine (ADMA), symmetric dimethylarginine (SDMA) and homoarginine (hArg): the ADMA, SDMA and hArg paradoxes.

Cardiovasc Diabetol 2018; 17:1.

* A review of the importance of homoarginine metabolism and its significance with regards to protein methylation, the concentration of methylated arginine in the plasma and the inhibition of NO synthesis.

[52] Kayacelebi AA, Langen J, Weigt-Usinger K et al. Biosynthesis of homoarginine (hArg) and asymmetric dimethylarginine (ADMA) from acutely and chronically administered free L-arginine in humans. Amino acids 2015; 47:1893-1908. 